Growth Curve Models in Retrospective Memory and Prospective Memory: The Relationship between Prediction and Performance with Task Experience

Amanda R. J. Ng ${ }^{\mathrm{a}}$, Romola S. Bucks ${ }^{\mathrm{a}}$, Shayne Loft ${ }^{\mathrm{a}}$, Steven P. Woods ${ }^{\mathrm{a}, \mathrm{b}}$, and Michael G. Weinborn $^{\mathrm{a}, \mathrm{c}}$

a School of Psychological Science

The University of Western Australia

35 Stirling Highway, Crawley WA 6009, Australia

${ }^{\mathrm{b}}$ Department of Psychology

The University of Houston

3695 Cullen Boulevard Room 126

Houston, TX 77204-5022, United States of America

${ }^{\mathrm{c}}$ School of Medicine and Health Sciences

Edith Cowan University

270 Joondalup Drive, Joondalup WA 6027, Australia

Corresponding Author:

Professor Romola S. Bucks

romola.bucks@uwa.edu.au / +61 864883267 (tel) / +61 864881006 (fax)

School of Psychological Science

The University of Western Australia

35 Stirling Highway, Crawley WA 6009, Australia

\title{
Disclosure of Interest
}

The authors report no conflicts of interest. This research did not receive any specific grant from funding agencies in the public, commercial, or not-for-profit sectors. 


\begin{abstract}
Older adults commonly experience declines in episodic memory that affect their daily lives. The aim was to examine whether the acquired metacognitive awareness that comes with task experience, indexed by meta-retrospective memory (meta-RM)/metaprospective memory (meta-PM), influences how older adults predict performance on later trials. Participants were 178 community-dwelling older adults. RM performance and predictions were measured using a multi-trial word-list learning task. Predictions and performance for PM were measured using a PM paradigm. Change in RM/PM performance and predictions over the trials/blocks were modelled using latent growth curve analyses. For RM, both predictions and performance increased with task experience. However, for PM, neither performance nor predictions changed with task experience. Hierarchical multiple regressions revealed that metacognitive awareness acquired during the RM and PM tasks influenced how older adults' modified their predictions of subsequent task performance. Findings are consistent with Toglia and Kirk’s (2000) hypothesis that individuals compare ongoing performance to expectations based on metacognitive knowledge, such that a discrepancy between actual and expected performance may influence emergent awareness.
\end{abstract}

Keywords: Metacognition; Prospective memory; Retrospective memory; Growth curves 
Metacognition refers to the knowledge and regulation of our cognition (Flavell, 1979), or simply "thinking about thinking". Since the term "metacognition" was first coined, there has been a proliferation of related terms: including, but not limited to, metacognitive knowledge, metacognitive awareness, metacognitive experience, and metamemory (Veenman, Van Hout-Wolters, \& Afflerbach, 2006). The term metamemory refers to our metacognition, or awareness, about our memory functioning. The first type of memory of interest in the current study is that of retrospective memory (RM). Meta$\mathrm{RM}$ is the process by which an individual retrospectively or prospectively makes judgments and assessments about their ability to recall or recognize past events (Dunlosky \& Metcalfe, 2008). The second type of memory of interest is prospective memory (PM). Meta-PM is the process by which an individual retrospectively or prospectively makes judgments and assessments about their ability to remember to perform deferred task actions (Einstein \& McDaniel, 2007). It is crucial to note that the execution of PM tasks requires both PM and RM (Kliegel, McDaniel, \& Einstein, 2000). The PM component involves remembering that you have to do something; the RM component involves remembering what the intended action is and when it should be done (e.g., buy bread when you pass the grocery store). RM is also needed to recall past performance (e.g., whether you were successful previously when you intended to buy bread). As such, awareness of performance of PM tasks involves awareness of PM abilities (meta-PM) and of RM abilities (meta-RM).

Metacognition is thought to be comprised of two components: knowledge about one's cognitive ability and monitoring of one's current cognitive performance (e.g., Flavell, 1979; Schraw \& Moshman, 1995; Schraw, Crippen, \& Hartley, 2006). The first component (metacognitive knowledge) is the overall understanding of one's own cognitive strengths and weaknesses, as well as internal and external factors (e.g., type of 
task, mood, past experience, etc.) that may affect cognition (Flavell, 1979; Lai, 2011). The second component (monitoring of cognition) is a dynamic process that involves one's own cognitive experiences (e.g., insights during the task, such as "I'm not doing that well”), identifying and selecting appropriate strategies, as well as setting and revising goals (Flavell, 1979; Schraw \& Moshman, 1995; Schraw et al., 2006; Whitebread et al., 2009). This monitoring of cognition is important for recognising inaccuracies or dissonance in task performance, and for the subsequent revision of goals and/or task expectations (i.e., learning from experience; Haller, Child, \& Wahlberg, 1988; Lai, 2011). These two components are not mutually exclusive: metacognitive knowledge facilitates cognitive monitoring and, in turn, metacognitive monitoring plays a role in the development and refinement of metacognitive knowledge (Lai, 2011).

Toglia and Kirk (2000) incorporated these two components of metacognition in a dynamic model of metacognitive awareness. They proposed two types of awareness: the first is intellectual awareness - an overall awareness of cognitive abilities independent of any specific task. The construct of cognitive knowledge comprises this type of awareness. The second is online awareness, which involves a judgment about a specific task and consists of anticipatory and emergent awareness. Anticipatory awareness is a person's expectations about how they might perform on a specific task, and is influenced by preexisting cognitive knowledge, beliefs and affect. Anticipatory awareness can be measured by obtaining predictions of task performance prior to acquiring task-specific experience. Emergent awareness involves the dynamic modification of anticipatory awareness based on task experience and is, likewise, influenced by factors such as cognitive knowledge, beliefs, and affect. In addition, it involves monitoring of the ongoing task, learning via feedback, adjustment of task expectations, modulating performance, and implementing strategies, as required. Performance predictions obtained after a task has begun, where 
there is more task-specific knowledge and opportunity to learn from task experience and adjust performance predictions, are measures of emergent awareness.

\section{Impact of Task Experience on Meta-Retrospective Memory Accuracy}

Given that emergent awareness, as defined in Toglia and Kirk’s (2000) model, develops with task-specific knowledge, feedback, and experience, it may be expected that participants would modify their performance predictions on subsequent performance in a task, such that RM performance predictions become more accurate with time. However, to our knowledge, there are no studies empirically testing if the metacognitive awareness acquired from RM performance does, indeed, influence how people make predictions on later blocks. In order to test this, one would need to quantify metacognitive awareness gained from an earlier trial of a task and test if it would significantly predict performance predictions (i.e., how participants expect they would perform) on the next trial of the same task. In the current study this was achieved by creating regression equations to predict performance predictions for each trial of a word-list learning RM task. These regression equations model the normative relationship between participants' predictions and performance and, therefore, allowed identification of what predictions (DV) a participant should have provided, given their performance (IV). The unstandardised residuals were extracted as indices of metacognitive accuracy for a particular trial. Residuals from the first trial represent anticipatory awareness, as predictions for this first trial were made before any specific task experience. Residuals from subsequent trials represent emergent awareness as, at each additional trial, participants are accumulating ongoing task experience and may modify their subsequent predictions as a result.

Consistent with the hypothesis that performance predictions would become more accurate over time, meta-RM studies have shown that relative accuracy (sensitivity for 
which items will be remembered versus which items will be forgotten) of meta-RM does improve with task experience. For example, participants' relative accuracy in judging how well they will learn and/or perform on episodic memory tasks improves across multiple trials (e.g., Ariel \& Dunlosky, 2011; Scheck \& Nelson, 2005; Serra \& Ariel, 2014; Serra \& Dunlosky, 2005; Tauber \& Dunlosky, 2015). However, absolute accuracy of metacognitive predictions does not appear to follow this same trajectory of improvement. Absolute accuracy is calculated by subtracting performance from prediction (prediction-performance discrepancies), where a zero value means absolute metacognitive accuracy, positive values indicate overconfidence, and negative values indicate underconfidence (e.g., Schraw, 2009). Studies have found a systematic bias in individuals' performance predictions on later trials: the underconfidence with practice (UWP) effect (e.g., Finn \& Metcalfe, 2007; Koriat, Sheffer, \& Ma’ayan, 2002; Serra \& Dunlosky, 2005). The UWP describes a shift from over-confident predictions to underconfident predictions that can occur as learning evolves (Koriat et al., 2002). For example, it is common for an individual to predict that they will perform slightly better than they actually score on the first trial of a word list learning task. On the second and subsequent trials of the word list-learning task, those individuals commonly shift toward predicting that they will perform slightly worse than they actually score. This UWP effect arises because participants' task performance (i.e., recall of words/items) improves with practice, but their corresponding RM predictions lag in comparison (Hanczakowski, Zawadzka, Pasek, \& Higham, 2013).

RM performance improves over repeated trials because participants are given multiple opportunities to encode and learn the same information (e.g., Karpicke \& Roediger, 2007; Jones et al., 2005; Koriat et al., 2002), but it is still not well understood how RM predictions change over time. That is, it remains unclear what drives the change 
(or lack of change) in meta-RM predictions over trials. As mentioned above, in accordance with Toglia and Kirk’s (2000) concept of online awareness, if participants are gaining metacognitive awareness through task experience, this should influence their predictions on later trials. For example if, in early trials, participants realise that the task is more difficult than expected and feel that they are not performing as well as they had anticipated, then they might lower their performance predictions at the next RM trial. This concept of online awareness has not previously been empirically tested. This would require measurement and statistical examination of whether acquired task experience on a previous task trial can influence meta-cognitive predictions on a following task trial. The novel data analysis strategy we detail above allows investigation of whether task experience, quantified by regression residuals representing metacognitive accuracy on earlier trials, would influence how participants predict their performance on subsequent trials. According to the definition of emergent awareness, participants should gain both task and performance-related information as the task progresses, which is likely to have an impact on their allocation of strategies and expectation of upcoming task performance. That is, this empirical test should demonstrate that acquired task experience will have a significant impact on later trial predictions.

As described, in detail below, the concept of online awareness is also potentially relevant to another type of commonly studied memory task that requires individuals to remember to perform deferred task actions in the future, that is PM.

\section{Impact of Task Experience on Meta-Prospective Memory Accuracy}

PM is the ability to remember to perform an intended action in the future, or remembering to remember. Meta-PM is an individual's metacognitive knowledge, or awareness, of their PM abilities (Einstein \& McDaniel, 2007). 
Researchers have studied meta-PM in clinical samples such as individuals with schizophrenia, human immunodeficiency virus (HIV), traumatic brain injury, and Parkinson’s disease (e.g., Casaletto et al., 2014; Knight, Harnett, \& Titov, 2005; Roche, Fleming, \& Shum, 2002; Smith, Souchay, \& Moulin, 2011). In general, these studies show that clinical populations have impairments in meta-PM often characterised by overconfidence in their PM abilities. Conversely, just five published studies have explored meta-PM in healthy adults (Meeks, Hicks, \& Marsh, 2007; Meier, von Wartburg, Matter, Rothen \& Reber, 2011; Rummel, Kuhlmann \& Touron, 2013; Schnitzspahn, Ihle, Henry, Rendell, \& Kliegel, 2011a; Schnitzspahn, Zeintl, Jager, \& Kliegel, 2011b). Methodological variations across the studies conducted with healthy adults likely explain a pattern of inconsistent results, with some studies reporting small to moderate levels of meta-PM accuracy (Meeks et al. 2007; Meir et al., 2011; Schnitzspahn et al., 2011b), while others report no relationship between prediction and performance (Rummel et al., 2013; Schnitzspahn et al., 2011a). Taken together, the data from this emerging literature show a small, but notable, positive relationship between PM performance and prediction, indicating that healthy adults have some level of awareness of their PM abilities. There is no evidence of an overall tendency for under- or overconfidence in this literature. To our knowledge, there are also no published papers investigating how meta-PM changes with experience on a PM task.

\section{The Present Study}

The aim of the study was to examine how participants' predictions and performance change with task experience on RM and PM tasks. The sample consisted of community-dwelling older adults. This is an important group in which to study both meta-RM and meta-PM as ageing brains are known to be particularly susceptible to both 
PM and RM failures, as well as reduced metacognitive awareness more generally (e.g., Connor, Dunlosky, \& Hertzog, 1997; Souchay, Isingrini, \& Espagnet, 2000). Given this, the use of an older adult sample was expected to provide us with the variability in metaPM, meta-RM and meta-memory required to conduct our individual differences analyses.

For the RM task, on the basis of the past literature, we predicted that 1) performance accuracy would improve with task experience, 2) RM predictions would increase with task experience but lag behind the increase in RM performance, and 3) there would be a small to medium positive relationship between RM predictions and performance. Finally, Toglia and Kirk’s (2000) concept of online awareness was empirically tested by examining whether the acquired metacognitive awareness that comes with ongoing task experience, indexed by meta-RM, significantly influences how participants predict performance on later trials.

As there are no studies investigating change in PM performance or predictions with task experience, the intention was to examine 1) how PM performance accuracy changes with task experience, and 2) how PM predictions change with task experience. Given the findings from prior research, it was predicted that 3) there would be a small to medium positive relationship between PM predictions and performance. In addition, Toglia and Kirk's (2000)'s concept of online awareness in meta-PM was tested by examining whether the acquired metacognitive awareness that comes with ongoing task experience, indexed by meta-PM, significantly influenced how participants predicted performance on later trials. 


\section{Method}

\section{Participants}

Participants aged 50 and above volunteered to participate from the West Australian Participant Pool and additional participants were also recruited from the Alzheimer's Australia Research Foundation WA Memory Study. All recruited participants were assessed within the Healthy Ageing Research Program (HARP) at the University of Western Australia. HARP is an observational, longitudinal study of cognitive aging amongst community-dwelling older adults. The measures used in the present study was embedded in a larger battery of neuropsychological measures that takes approximately 3 hours. Based on a self-report medical history questionnaire, participants with severe neurological (e.g., stroke, $n=7$ ) or psychiatric conditions (e.g., schizophrenia, $n=2)$ or previous loss of consciousness of more than 30 minutes $(n=4)$ were excluded. A further 5 participants were excluded due to large amounts of missing data. The final sample consisted of 178 participants (62.9\% female), with a mean age of 71.56 years (SD $=7.69 ; 55$ to 93 years), and 13.59 mean years of education $(\mathrm{SD}=3.43) ; 174(97.8 \%)$ participants identified as Caucasian.

\section{Materials and Procedure}

Retrospective memory task. RM was measured using the word list recall subtest of the Repeatable Battery for the Assessment of Neuropsychological Status - Form A (RBANS; Randolph, Tierney, Mohr \& Chase, 1998). RBANS has been studied relatively extensively in normal samples, and has been demonstrated to be useful in the assessment of neuropsychological functioning in various clinical conditions (e.g., Dickerson et al., 2004). The word list recall subtest on the RBANS shows good convergent validity with other tests of RM such as the California Verbal Learning Test Second Edition $(r=.70$; 
McKay, Casey, Wertheimer, \& Fichtenberg, 2007). The RBANS requires participants to read a list of 10 words, and then recall as many words as they can from the word list, in any order. The list was then reread in the same order, with participants recalling the words after each reading, for a total of four trials. RM performance for each trial was recorded out of 10 (e.g., 4 out of 10) and was converted into percentage (e.g., 40\%), for consistency with the PM performance measure (Table 2).

Assessment of meta-retrospective memory. To assess meta-RM, participants predicted their performance on each of the RBANS word list recall subtests. Before each of the four readings of the word list, participants were first told that they would be read a list of 10 words. They were then asked, "Before we begin, how many words do you think you will remember”. RM prediction for each trial was recorded out of 10 (e.g., participant said they would get 4 out of 10 words) and was converted into percentages (e.g., $40 \%$ prediction).

Prospective memory task. A variant of the Einstein and McDaniel (1990) paradigm, with a non-focal PM task embedded in an ongoing word categorisation task, was used in this study. Participants responded using the computer keyboard.

The ongoing task involved participants reading word pairs and indicating as quickly and as accurately as possible whether a lower-case word on the left side of the screen belonged to the category described by the upper-case word on the right (Einstein et al., 2005). Participants were told to indicate as quickly and accurately as possible whether the word on the left of the screen was a match (e.g., eagle-BIRD) or non-match (e.g. owlFURNITURE), with the category word on the right. Participants indicated this by pressing the "l” key, labelled with a "Y", for a match, and the "a” key, labelled with an “N”, for a non-match. On each trial, a blank screen was first presented for $250 \mathrm{~ms}$, followed by a fixation cross for 250ms; a word-category pair was then presented. The 
presentation of the next trial was triggered by the participant's response or after $5000 \mathrm{~ms}$, whichever occurred first. The PM task required the participants to press the "6" key, labelled with an "X”, whenever they saw the syllable "tor", instead of responding to the ongoing task.

Two 400-word-pair sets were created from an updated version of the Battig and Montague (1969) category norms (Van Overschelde, Rawson \& Dunlosky, 2004), one set required the "Yes" (match) response and the other required the "No" (non-match) response; 24 additional words were used as non-focal PM cues “tor” (e.g., tornado, motorcycle, doctor), of which 12 words matched the category they were paired with, and the other 12 were non-match. The two, 400-word pair sets were randomly split into four blocks (baseline and three PM blocks), and each block consisted of 100 matched wordcategory pairs and 100 non-matched pairs. Twelve matched PM word-pairs and 12 nonmatched PM word-pairs were randomly split into three blocks (three PM blocks), such that each PM block would have four matched and four non-matched PM word-category pairs. The ongoing task word sets and PM word sets were counterbalanced, and the order of trials within each block was randomised. No PM targets were presented within the first five trials of each block, after which the eight PM targets per block were distributed randomly within every 25 trials, with at least two ongoing task trials between PM targets.

In order to include a measure of baseline category decision performance, we presented a baseline block before the PM blocks. All participants received instructions on the word-categorisation task, followed by six practice trials. Participants received accuracy and speed feedback during the practice trials, and if they failed the first set of practice trials (fewer than 4 out of 6 correct), they were given another set of six practice trials. All participants passed either the first or second set of practice trials. In the baseline block, participants performed baseline categorisation trials. After this baseline block, 
participants were informed that we were also interested in their ability to remember to perform actions in the future, and the PM task was introduced. After participants received instructions on the PM task, they completed another 10 practice trials with the PM target appearing in the seventh item. Similar to the baseline practice trials, participants received accuracy and speed feedback on the PM practice trials. No feedback was provided during the test blocks. To check that PM failures were not because participants did not understand the task, all participants were asked to clarify both PM and ongoing task instructions upon completion of each PM block: all participants accurately recalled these instructions. A total of three PM blocks was administered. Each PM block consisted of 200 trials, eight of which were PM targets. Participants were not told how many trials in total or how many PM targets to expect in each block. Before each PM block, participants were reminded that their goals were to try their best at both the ongoing and the PM task, and they then completed a two-minute distractor Sudoku puzzle. Upon completion of each PM block, participants were asked to clarify both PM and ongoing task instructions. PM performance was recorded as a percentage (Table 2).

Assessment of meta-prospective memory. Participants predicted their performance for both the ongoing and the PM task, in order to avoid participants concluding that the PM task was of greater importance than the ongoing task. They were asked the following questions before each block of the experiment and prior to the distractor task: "What percentage of words will you judge correctly as belonging, or not, to a category", and "What percentage of tor syllables will you respond to successfully?" Participants were given a visual analogue scale (0\% to 100\%) to indicate their response. PM predictions for all three blocks are shown in Table 2. 


\section{Analytic Approach}

Growth Curves. Individual differences in $\mathrm{PM}$ and $\mathrm{RM}$ performance and prediction trajectories were analysed using latent growth curve modelling (LGM; Duncan, Duncan, Strycker, Li, \& Alpert, 1999; Muthén, 1991). Analyses were conducted in MPlus version 7.4 (Muthén \& Muthén, 1998 - 2010).

Based on the raw data, different functional forms for RM and PM growth curves were considered, including intercept-only, linear, quadratic, and logarithmic growth trends. For RM, we also estimated a model with the $2^{\text {nd }}$ and $3^{\text {rd }}$ trial freely estimated for performance data and another 'approximately logarithmic' model for both performance and prediction data. The time vector (i.e., repeated blocks $t=1,2,3$ for PM and repeated trials $t=1,2,3,4$ for $R M$ ) was scaled such that the final time step was equal to 1 to facilitate the interpretation of the estimated mean for the change across trials (e.g. 0, 0.5, 1, and $0,0.33,0.67$, and 1.0 for RM and PM linear growth, respectively). Solutions obtained for models of different functional forms were compared by visually inspecting plots of fitted and observed means, and by comparing fit indices: chi-square $\left(\chi^{2}\right)$, Akaike Information Criterion (AIC; Akaike, 1974), Comparative Fit Index (CFI; Bentler, 1990), Tucker/Lewis Index (TLI; Tucker \& Lewis, 1973) and the Root Mean Square Error of Approximation (RMSEA; Browne \& Cudek, 1992). We report a range of fit statistics: CFI and TLI values above .90 indicate reasonable fit, and above .95 good fit; RMSEA values $<.05$ indicate good fit, whilst RMSEA values with $90 \%$ confidence intervals that cross .05 are acceptable; SRMR values $<.08$ indicate good fit (Marsh, Hau, \& Wen, 2004). Given the known sensitivity of chi-square to sample size (Hooper, Coughlan, \& Mullen, 2008), chi-square adjusted for degrees of freedom ( $\left.\chi^{2} / \mathrm{df}\right)$ was calculated, where reasonable fit is indicated when the value is < 3 (Hu \& Bentler, 1999). Model parsimony was summarised with the parsimony ratio (Raykov, Tomer, \& Nesselroade, 1991). 
After suitable growth models for RM prediction and RM performance were developed, we evaluated the relationship between PM prediction and performance by combining the two individual growth models. The same method was followed to investigate the relationship between PM prediction and performance.

Test of Anticipatory and Emergent Awareness. Three regression equations were formulated to test the concept of online (anticipatory and emergent) awareness (Table 1). The regression equations seek to model the change in participants' predictions of how well they will do as they gain task experience.

Here we use PM as an illustrative example (the same steps were applied to RM variables). A linear regression was first used to predict Block 1 PM predictions by regressing Block 1 PM predictions onto Block 1 PM performance, including age and education as covariates. The unstandardised residuals from this equation, calculated as actual prediction minus predicted prediction, reflect anticipatory awareness as participants made their predictions prior to any task specific experience. The residuals represent the deviation from what is normal metacognition, such that a residual of zero indicates normatively accurate metacognition and non-zero residuals indicate metacognitive inaccuracy.

A hierarchical regression analysis was used to predict Block 2 PM predictions. At the first step, Block 2 PM performance, age, and education were entered into the model as predictors of Block 2 PM predictions. At the second step, anticipatory awareness was added as metacognitive knowledge gained from the first round of task experience should, in theory, contribute to how individuals predict their performance on the next block. The unstandardised residuals from this equation reflect early emergent awareness as participants made Block 2 predictions after acquiring task experience from Block 1. 
A final hierarchical regression analysis was used to predict Block 3 PM predictions. At the first step, Block 3 PM performance, age, and education were entered into the model as predictors of Block 3 PM predictions. At the second step, anticipatory awareness was added in. At the third and final step, early emergent awareness was included, as the additional metacognitive knowledge from Block 2 may also impact on an individuals' prediction of the final PM block. The unstandardised residuals from this equation reflect late emergent awareness as participants made Block 3 predictions after acquiring task experience from both Blocks 1 and 2.

\section{Results}

\section{Retrospective Memory}

Retrospective memory performance. For RM performance, following formal indicators of model fit, the best fitting model described a logarithmic growth curve. The parameter estimates for this final logarithmic growth model are provided in Supplementary Table 1 . Significant intercept ${ }^{\mathbf{1}}$ and slope indicate that an initial level of RM performance was identified, and participants improved in their performance over trials (slope was positive). Significant individual variability for both the initial performance factor and the logarithmic change factor were detected. Finally, initial RM performance and change were not significantly correlated (model-implied correlation -

\footnotetext{
${ }^{1}$ Latent growth curve analysis seeks to model an intercept term representing the mean starting value of the sample as a whole. Variability around this intercept is also modelled. In an intercept-only model, the intercept represents the mean value of the variable being modelled. In growth curve models (e.g., linear, quadratic, logarithmic), the intercept represents the average initial value being modelled and the slope term(s) represents the average rate of change in the variable. It is possible for the intercept term to be nonsignificant, but for there to be significant variability. This would indicate that no average initial value could be found and that the initial values in the sample were highly variable. Likewise, a slope term can be nonsignificant with significant variance, indicating no average change, but significant variability in the slope of change over time. This variability around the intercept and slope terms reflects individual differences in initial performance and change in performance over time.
} 
$.14, p=.556)$; that is, there was no relationship between initial performance and subsequent performance improvement over trials.

Retrospective memory predictions. Following formal indicators of model fit, the quadratic model was chosen as the best fitting model for RM predictions. The parameter estimates for this quadratic growth model are provided in Supplementary Table 1. A significant intercept indicates that an initial level of RM prediction was identified. The slope was positive, indicating that participants increased their predictions over the four trials. The quadratic term was non-significant, which suggests that participants' RM predictions trajectory do not follow a quadratic growth function. However, the significant variance associated with the slope and quadratic terms indicates that there are two sources of variability associated with the increase in $\mathrm{RM}$ predictions. Larger $\mathrm{RM}$ prediction slopes of change were significantly associated with a smaller quadratic function (modelimplied correlation was -.86, $p<.001)$ : that is, those who started out with higher rates of increase in prediction, reached asymptote faster as their rate of change was slower at the end. Finally, initial RM prediction and slope were not significantly correlated (modelimplied correlation $-.22, p=.350$ ) and neither were initial RM prediction and quadratic growth function (model-implied correlation .16, $p=.530$ ); participants' predictions at Trial 1 had no relationship with their rate of change in predictions over time. 
Relationship between retrospective memory prediction and performance. To understand the relationship between RM prediction and performance, the logarithmic RM performance model and quadratic RM prediction models were combined. Model fit indices and standardised model estimates are shown in Supplementary Table 1. Higher initial RM performance was associated with higher initial RM prediction $(r=.38, p=$ .007), suggesting that those who predicted they would do well were more likely to do so. High initial RM performance was also associated with a larger slope of change in RM prediction ( $r=.58, p=.003$ ) but with a smaller quadratic growth function of RM prediction $(r=-.35, p=.034)$, indicating that participants who performed better initially increased their predictions over the four trials, but with increasingly smaller rates of increase with each subsequent trial (i.e., the magnitude of prediction increase plateaued).

The RM prediction intercept was larger than the performance intercept, suggesting that, at Trial 1, participants overestimated their performance $\left(\chi^{2}\right.$ diff $\left.=6.46, p=.011\right)$. However, the slope of RM performance was larger than that of prediction, indicating that there was greater improvement in performance compared to increase in predictions. In other words, predictions lagged behind performance $\left(\chi^{2}\right.$ diff $\left.=19.55, p<.001\right)$.

Retrospective memory anticipatory and emergent awareness. Linear regression analysis was used to predict Trial 1 RM prediction from RM performance, age, and years of education. This yielded a non-significant (Trial 1 Model 1; Table 3), $p=$ .091. Only $3.8 \%$ of the variance in Trial 1 RM predictions was accounted for by the predictors in the model. Trial $1 \mathrm{RM}$ performance predicted Trial $1 \mathrm{RM}$ predictions at trend level $(p=.051)$. The unstandardised residuals from this regression equation were then saved as a representation of anticipatory awareness.

Hierarchical multiple regression analysis was used to test if Trial 2 RM performance, age, years of education, and anticipatory awareness significantly predicted 
Trial 2 RM predictions. This yielded a statistically significant model (Trial 2 Model 2; Table 3) $R^{2}=.31, R^{2}$ Adjusted $=.30, F(4,169)=18.75, p<.001 ; 31.2 \%$ of the variance in Trial 2 RM predictions was accounted for by the predictors in the model, and $\mathrm{R}^{2}$ change was .16, indicating that $16 \%$ additional variance in Trial 2 RM predictions was explained by adding anticipatory awareness. Trial 2 RM performance $(p<.001)$ and anticipatory awareness $(p<.001)$ significantly predicted Trial 2 RM predictions, with greater discrepancies indicative of overconfidence associated with higher Trial 2 predictions. The unstandardised residuals from this regression equation were saved as a representation of early emergent awareness.

Next, Trial 3 RM performance, age, years of education, anticipatory awareness, and early emergent awareness were tested as predictors of Trial 3 RM predictions. This yielded a statistically significant model (Trial 3 Model 3; Table 3) $R^{2}=.61, R^{2}$ Adjusted $=$ $.59, F(5,169)=50.55, p<.001$. Thus, $61 \%$ of the variance in Trial 3 RM predictions was accounted for by the predictors in the final model; inspection of model 2 and 3's $R^{2}$ change values indicate that an additional $4.9 \%$ variance in Trial 3 RM predictions was provided when adding anticipatory awareness, and another $27 \%$ when adding early emergent awareness. Anticipatory awareness $(p<.001)$ and emergent awareness $(p<$ .001) significantly predicted Trial 3 RM predictions, again, with greater discrepancies indicative of overconfidence for both Trials 1 and 2 associated with higher predictions for Trial 3. The unstandardised residuals from this regression equation were saved as a representation of mid-emergent awareness.

Finally, Trial 4 RM performance, age, years of education, anticipatory awareness, early emergent awareness, and mid-emergent awareness were tested as predictors of Trial 4 RM predictions. The results yielded a statistically significant model (Trial 4 Model 3; Table 3) $R^{2}=.67, R^{2}$ Adjusted $=.65, F(6,169)=53.62, p<.001 ; 66.5 \%$ of the variance in 
Trial 4 RM predictions was accounted for by the predictors in the final model, and model 2 to 4 's $\mathrm{R}^{2}$ change values show that an additional 3.3\% variance in Trial 4 RM predictions was provided when adding anticipatory awareness, another $18.5 \%$ when adding early emergent awareness, and yet another $1.2 \%$ when adding mid emergent awareness. Anticipatory awareness $(p<.001)$, early emergent awareness $(p<.001)$, and mid emergent awareness $(p<.001)$ significantly predicted Trial 4 RM predictions. The pattern of predictions was identical to that above.

Retrospective memory summary. Our findings demonstrate that, in line with hypothesis 1, RM performance increased over trials in a logarithmic fashion. Hypothesis 2 was also supported: RM predictions increased, albeit in a quadratic curve function, and were noted to lag behind performance. A medium, positive relationship between initial RM predictions and performance was found, supporting hypothesis 3. Finally, in our empirical test of Toglia and Kirk’s (2000) concept of online awareness, we found that the acquired metacognitive awareness that comes with ongoing task experience, indexed by meta-RM, significantly influenced how participants predicted performance on later trials, with greater overconfidence on earlier trials being associated with higher predictions on subsequent trials.

\section{Prospective Memory}

Prospective memory performance. Whilst a linear growth curve model was estimated to explore if there was linear change in PM performance over the three blocks, and despite good fit, it was not substantially better than the more parsimonious, interceptonly model (see Supplementary Table 2). The significant intercept means that a mean level of overall PM performance was identified as 64.51\% accuracy. However, there was also a significant amount of variance around this intercept which indicated that there were 
individual differences in participants' overall performance on the PM task. No slope (or slope variance) was identified, indicating that there was no change in PM performance over the blocks.

Prospective memory predictions. For PM predictions, an intercept-only model was first estimated, which fit very poorly, followed by a linear growth curve model. The latter model represented a substantial improvement in model fit; the parameter estimates are provided in Supplementary Table 2. An initial level of PM prediction was identified (significant intercept) but there were also individual differences around how participants predicted their PM performance at baseline (significant variance of intercept). The slope was not significant but there was substantial variance, indicating that, although, on average, participants did not change their PM predictions over blocks, there was marked variability in how some individuals modified their PM predictions in subsequent blocks. Some increased their predictions, some reduced them, and some did not change their predictions from block to block. Further analyses investigating this variation in the PM prediction slope are reported below. There was no relationship between initial PM prediction and the slope of change (model-implied correlation -.05, $p=.720$ ). 
Relationship between prospective memory predictions and performance. To understand the relationship between PM prediction and performance, the intercept-only PM performance model and linear PM prediction model were combined (Supplementary Table 2). Participants who predicted that they would do well on the PM task did, indeed, perform better, $r=.30, p=.001$. However, there was no relationship between initial PM performance and change in PM prediction, $r=.04, p=.720$; whether or not participants performed well or poorly in the first PM block did not relate to how they modified their performance expectations on subsequent blocks.

Prospective memory anticipatory and emergent awareness. Linear regression analysis was used to predict Block 1 PM prediction from PM performance, age, and years of education. The results yielded a model with trend-level significance (Block 1 Model 1; Table 4) $R^{2}=.04, R^{2}$ Adjusted $=.03, F(3,173)=2.54, p=.058$. Only $4 \%$ of the variance in Block 1 PM predictions was accounted for by the predictors in the model. Block 1 PM performance $(\beta=.18, p=.020)$ significantly predicted Block 1 PM predictions. Unstandardised residuals were saved to index anticipatory awareness.

Hierarchical multiple regression analysis was used to test if Block 2 PM performance, age, years of education, and anticipatory awareness (Block 1 residuals) significantly predicted participants' Block 2 PM predictions. The results yielded a statistically significant model (Block 2 Model 2; Table 4) $R^{2}=.44, R^{2}$ Adjusted $=.42, F(4$, $173)=32.49, p<.001 .44 \%$ of the variance in Block 2 PM predictions was accounted for by the predictors in the model; $R^{2}$ change was .39, indicating that of this $44 \%, 39 \%$ additional variance in Block 2 PM predictions was provided by adding anticipatory awareness. As shown in Table 4, Block 2 PM performance $(\beta=.17, p=.007)$ and anticipatory awareness $(\beta=.62, p<.001)$ significantly and positively predicted Block 2 PM predictions. That is, overconfidence in Block 1 was associated with higher 
predictions of PM performance in Block 2. Unstandardised residuals were saved to index early emergent awareness.

Hierarchical regression analysis was again used to test if Block 3 PM performance, age, years of education, anticipatory awareness, and early emergent awareness significantly predicted participants' Block 3 PM predictions. The results yielded a statistically significant model (Block 3 Model 3; Table 4) $R^{2}=.68, R^{2}$ Adjusted $=$ $.67, F(5,173)=71.29, p<.001$. Thus, $68 \%$ of the variance in Block 3 PM predictions was accounted for by the predictors in the final model; inspection of model 2 and 3's $R^{2}$ change values indicated that an additional 36\% variance in Block 3 PM predictions was provided when adding anticipatory awareness, and another $27 \%$ when adding early emergent awareness. Anticipatory awareness $(\beta=.62, p<.001)$ and early emergent awareness $(\beta=.53, p<.001)$ significantly predicted Block 3 PM predictions. As with Block 2, those with greater overconfidence on Blocks 1 and 2, predicted their Block 3 performance as being higher.

Prospective memory summary. Our findings demonstrate that, at a group level, there was no overall tendency for an increase or decrease in PM performance or PM predictions with task experience. However, significant individual differences in the PM prediction slope indicated that, while the overall group's PM predictions did not change, there appeared to be differences in how individual participants adjusted their predictions (i.e., some participants increased their predictions while others decreased them with task experience). A small, positive relationship between overall PM predictions and performance was found. Finally, in our empirical test of Toglia and Kirk’s (2000) concept of online awareness, we found that acquired metacognitive awareness that comes with ongoing task experience, indexed by each individual's meta-PM (residual) score, was significantly related to participants' predictions of their performance on later trials. That 
is, individuals who had larger discrepancies between prediction and performance reflective of overconfidence were more likely, on subsequent trials, to say they would do better.

\section{Discussion}

This study's aim was to characterise both meta-prospective (meta-PM) and metaretrospective memory (meta-RM) by using latent growth curve modelling to investigate how predictions and performance change with task experience on RM and PM tasks, in a sample of community-dwelling older adults. The effect of Toglia and Kirk’s (2000) concept of anticipatory and emergent awareness - that is the potential benefit of task experience - in both RM and PM was also tested.

In doing so we have introduced two novel approaches to the analysis of meta-PM and meta-RM: latent growth curve modelling of prediction and performance, and the new regression method to model the normative relationship between participants' predictions and performance. Prior studies have utilised analysis of variance in their statistical analysis. Analysis of variance provides an answer only to the question of whether (or not) there are differences in mean scores, for the participants as a whole, and whether those means vary across blocks. It does not give information about the direction of those differences and it assumes that the differences between blocks occur in a generally linear fashion. In ANOVA analysis, by definition, one can analyse only prediction or performance or a simple discrepancy between them. By contrast, the more sophisticated latent growth curve modelling method allowed us to model how prediction and performance change at the level of the individual, which allowed consideration of whether the general change in prediction/performance across blocks was linear or some other function. Critically, we then used the change scores (slope terms) derived from the 
analyses as dependent or predictor variables so that we could see the extent to which they were related to each other, and/or as a function of baseline (intercept) scores. This novel analytic technique is powerful because it allows exploration of individual differences in performance and the associates of such individual differences.

Our second, novel analytic approach regressed performance on prediction to generate unstandardised residuals to be used as indices of metacognitive accuracy. This method contextualises metacognitive accuracy within what is normal for the sample and partials out error variance from the index of metacognitive accuracy. This method, too, allows exploration of individual differences and, critically, of the effect of differences between performance and prediction at one block or trial on subsequent blocks or trials.

\section{Retrospective Memory Predictions and Performance}

Although several studies have explored meta-RM, to our knowledge none has looked at RM predictions and performance separately. Consistent with the existing literature in older adults (e.g., Jones et al., 2005; Koriat et al., 2002; Tauber \& Rhodes, 2012), RM performance improved with experience. Similarly, participants increased their RM predictions with task experience. RM predictions started off higher than performance (overconfidence) but then increased at a slower rate compared to performance. These findings are consistent with the underconfidence-with-practice effect (e.g., Finn \& Metcalfe, 2007), which states that people typically start off slightly overconfident and become increasingly more underconfident with task experience due to smaller increases in predictions compared to actual performance.

The combined model of RM prediction and performance showed that those who said they would do better on initial list recall did so. This finding is consistent with the wider RM literature (e.g., Connor et al., 1997; Hertzog, Dixon, \& Hultsch, 1990; 
McDonald-Miszcazk, Hunter, \& Hultsch, 1994; Wood et al., 2008). Change in RM predictions with task experience was not associated with change in RM performance over time, again, providing further evidence that participants' predictions do not follow the same improvement trajectory as their performance (e.g., Finn \& Metcalfe, 2007).

\section{Prospective Memory Predictions and Performance}

We found that, at the group level, neither PM performance nor PM predictions changed with task experience. However, significant individual differences in the prediction slope term suggested that there appeared to be differences in how participants adjust their PM predictions with task experience. The combined model of PM prediction and performance was estimated to investigate the relationship between the two, revealing that although overall PM performance was not related to PM prediction change over blocks, overall PM prediction was significantly associated with PM performance. Those who said they would do better did, indeed, have better performance. This relationship between PM performance and prediction suggests that participants appear to have some level of awareness of their PM abilities but, given the small effect, there may be other variables such as cognitive complaints, objective cognition (e.g., executive functions) or mood that affect this relationship.

\section{Anticipatory and Emergent Awareness in Retrospective and Prospective Memory}

To our knowledge, this is the first study to have examined if anticipatory and emergent awareness - that is, the potential benefit from task experience, would contribute to subsequent predictions of performance. Toglia and Kirk (2000) argue that in online awareness, acquired metacognitive awareness through task experience will impact on later awareness. Our results are consistent with Toglia and Kirk’s (2000) model of metacognitive awareness, which postulates that individuals compare ongoing 
performance to expectations based on metacognitive knowledge, such that a discrepancy between actual and expected performance may influence emergent awareness. We showed that anticipatory awareness, indexed by Trial 1 meta-RM (operationalised as performance-prediction residuals), was a significant predictor of participants' predictions for Trial 2. Emergent awareness, which is acquired while obtaining task-specific experience, was indexed by Trial 2 meta-RM (early emergent awareness) and Trial 3 meta-RM (late emergent awareness). Anticipatory and early emergent awareness were both significant predictors of Trial 3 predictions, whilst anticipatory, early, and late emergent awareness predicted participants' final trial (Trial 4) predictions. Similar results were found for PM, where anticipatory and emergent awareness, as indexed by meta-PM, were significant predictors of how participants predicted subsequent trials.

This study presents the first empirical test of Toglia and Kirk’s (2000) concept of online awareness. The findings suggest that pre-existing knowledge or beliefs about a specific task (anticipatory awareness) influence a person's expectations about how they will perform on a task they are yet to experience. Once task-specific experience has been acquired, this anticipatory awareness is modified by factors such as task monitoring, internal or external performance feedback, and use of strategies. This dynamic change in task awareness likely reflects emergent awareness and, as stated in Toglia and Kirk's (2000) model, may be influenced by cognitive factors, beliefs regarding task and self, and affect. As our data show, as more task experience is acquired, emergent awareness grows, resulting in the modification of performance expectations (i.e., predictions of performance). 


\section{Limitations and Future Research Directions}

A limitation of this study is that the only covariates explored in this relationship were age and years of education. Toglia and Kirk (2000) also proposed that factors such as emotional states, knowledge of task, task difficulty, and cognition contribute to online awareness. Future studies may wish to model the impact of such covariates when exploring online awareness.

A defining feature of PM tasks is that, in contrast to RM tasks, individuals are not explicitly directed when to engage in memory retrieval. Instead, prospective remembering requires individuals to self-initiate the recollection of intended actions in response to PM cues that are also appropriate cues for more routine, ongoing task responses (Heathcote, Loft, \& Remington, 2015; Strickland, Loft, Remington, \& Heathcote, in press). As a consequence, RM performance can be more easily monitored. In the current study, participants studied 10 words and knew how many words they recalled. In contrast, participants were not made aware how many PM targets they would encounter. To some extent, this is also likely true in daily life. RM error monitoring is likely easier because we are in a retrieval mode (recalling a shopping list). For PM tasks we may not realize we have encountered the relevant PM retrieval occasion (driving past the pharmacy but not stopping to get medication). It would be useful for future research to tease apart the effect of some of the differences between RM tasks and PM tasks on metacognition.

More generally, care must be taken in generalising our findings to everyday life because the types of PM and RM tasks encountered in everyday life are, undoubtedly, more nuanced and complex than the highly controlled RM and PM tasks we used. For example, in everyday life people may form an intention for a given day and then have to re-evaluate the likelihood that the intention can be completed based on other obligations that arise during the day. Moreover, daily life further complicates the picture as the 
complexity of the ongoing tasks can wax and wane, people can use various compensatory strategies, motivational issues come into play, and there are a host of clinical factors (e.g., medical, psychiatric, and psychosocial) that can influence PM and RM in these settings (see Casaletto et al., 2017). It may be challenging to study meta-PM using more naturalistic PM tasks, but it may be a potentially interesting extension to this line of research.

Finally, this study reports metamemory in an older adult sample. We wished to determine the relationships between prediction and performance, at the level of the individual, for which we needed to be confident that there would be sufficient variability in performance. Accordingly, we chose an older adult sample because PM, RM and metamemory have been shown to decline with age, but with marked individual variability in the degree of age-related change. Now that the methods present in the current paper have been established as valid and reliable, it will be important to determine if the same patterns are found in younger adults and to compare older and younger adults.

\section{Conclusion}

Our ability to learn from experience plays an important role in improving future performance. Everyday memory tasks require both RM and PM. In particular, many everyday activities important to older adults' health and safety, such as managing complex medication regimens or attending doctor's appointments, are heavily reliant on RM and PM abilities. As such, it is important to understand how individuals are able to draw on past successes or failures in such tasks and, subsequently, improve performance and adjust their metacognitive awareness of their abilities. Having a better understanding of older adults' awareness of their RM and PM abilities can provide insight into the extent to which they would use internal or external strategies to compensate for memory 
deficits, or be willing to participate and/or able to benefit from memory intervention programs. Our novel data analysis strategy has provided direct support for Toglia and Kirk's concept of online awareness that metacognitive awareness acquired by ongoing task experience helps shape predictions of later tasks. Further studies investigating factors such as affect, cognition, and task knowledge on meta-PM will be necessary. 


\section{References}

Akaike, H. (1974). A new look at the statistical model identification. IEEE transactions on automatic control, 19(6), 716-723.

Ariel, R., \& Dunlosky, J. (2011). The sensitivity of judgment-of-learning resolution to past test performance, new learning, and forgetting. Memory \& Cognition, 39(1), 171-184.

Battig, W. F., \& Montague, W. E. (1969). Category norms of verbal items in 56 categories A replication and extension of the Connecticut category norms. Journal of Experimental Psychology, 80(3p2), 1.

Bentler, P M. (1990). Comparative fit indexes in structural models. Psychological Bulletin, 107, 238-246.

Browne, M. W., \& Cudeck, R. (1992). Alternative ways of assessing model fit. Sociological Methods \& Research, 21(2), 230-258.

Casaletto, K. B., Doyle, K. L., Weber, E., Woods, S. P., Heaton, R. K., Grant, I., ... \& Marquie-Beck, J. (2014). Self-predictions of prospective memory in HIVassociated neurocognitive disorders: evidence of a metamemory deficit. Archives of Clinical Neuropsychology, 29(8), 818-827.

Casaletto, K.B., Weber, E., Iudicello, J.E., \& Woods, S.P. (2017). Real-world impact of HIV-associated neurocognitive impairment. In N. Chiaravalloti and Y. Goverover (Eds.), Changes in the Brain, Impact Daily Life. Pp. 211-245. New York: Springer. 
Connor, L. T., Dunlosky, J., \& Hertzog, C. (1997). Age-related differences in absolute but not relative metamemory accuracy. Psychology and Aging, 12(1), 50.

Dickerson, F., Boronow, J. J., Stallings, C., Origoni, A. E., Cole, S. K., \& Yolken, R. H. (2004). Cognitive functioning in schizophrenia and bipolar disorder: comparison of performance on the Repeatable Battery for the Assessment of Neuropsychological Status. Psychiatry research, 129(1), 45-53.

Duncan, T. E., Duncan, S. C., Strycker, L. A., Li, F., \& Alpert, A. (1999). An introduction to latent variable growth curve modeling: Concepts, issues, and application. Mahwah NJ: Lawrence Erlbaum Associates.

Dunlosky, J., \& Metcalfe, J. (2008). Metacognition. Sage Publications.

Einstein, G. O., \& McDaniel, M. A. (1990). Normal aging and prospective memory. Journal of Experimental Psychology: Learning, Memory, and Cognition, 16(4), 717.

Einstein, G. O., \& McDaniel, M. A. (2007). Prospective memory and metamemory: The skilled use of basic attentional and memory processes. The Psychology of Learning and Motivation, 48, 145-173.

Finn, B., \& Metcalfe, J. (2007). The role of memory for past test in the underconfidence with practice effect. Journal of Experimental Psychology: Learning, Memory, and Cognition, 33(1), 238.

Finn, B., \& Metcalfe, J. (2008). Judgments of learning are influenced by memory for past test. Journal of memory and language, 58(1), 19-34. 
Flavell, J. H. (1979). Metacognition and cognitive monitoring: A new area of cognitive-developmental inquiry. American Psychologist, 34(10), 906-911.

Haller, E. P., Child, D. A., \& Walberg, H. J. (1988). Can comprehension be taught? A quantitative synthesis of metacognitive studies. Educational Researcher, 17(9), 5-8.

Hanczakowski, M., Zawadzka, K., Pasek, T., \& Higham, P. A. (2013). Calibration of metacognitive judgments: Insights from the underconfidence-with-practice effect. Journal of Memory and Language, 69(3), 429-444.

Heathcote, A., Loft, S., \& Remington, R. W. (2015). Slow down and remember to remember! A delay theory of prospective memory costs. Psychological Review, 122, 376-410.

Hertzog, C., Dixon, R. A., \& Hultsch, D. F. (1990). Relationships between metamemory, memory predictions, and memory task performance in adults. Psychology and Aging, 5(2), 215.

Hooper, D., Coughlan, J., \& Mullen, M. (2008). Structural equation modelling: Guidelines for determining model fit. Articles, 2.

Hu, L. T., \& Bentler, P. M. (1999). Cutoff criteria for fit indexes in covariance structure analysis: Conventional criteria versus new alternatives. Structural equation modeling: a multidisciplinary journal, 6(1), 1-55.

Jones, R. N., Rosenberg, A. L., Morris, J. N., Allaire, J. C., McCoy, K. J., Marsiske, M., ... \& Malloy, P. F. (2005). A growth curve model of learning acquisition among cognitively normal older adults. Experimental Aging Research, 31(3), 291-312. 
Karpicke, J. D., \& Roediger, H. L. (2007). Repeated retrieval during learning is the key to long-term retention. Journal of Memory and Language, 57(2), 151-162.

Kliegel, M., McDaniel, M. A., \& Einstein, G. O. (2000). Plan formation, retention, and execution in prospective memory: A new approach and age-related effects. Memory \& cognition, 28(6), 1041-1049.

Knight, R. G., Harnett, M., \& Titov, N. (2005). The effects of traumatic brain injury on the predicted and actual performance of a test of prospective remembering. Brain Injury, 19(1), 19-27.

Koriat, A., Sheffer, L., \& Ma'ayan, H. (2002). Comparing objective and subjective learning curves: judgments of learning exhibit increased underconfidence with practice. Journal of Experimental Psychology: General, 131(2), 147.

Lai, E. R. (2011). Metacognition: A literature review. Always learning: Pearson research report.

Marsh, H. W., Hau, K. T., \& Wen, Z. (2004). In search of golden rules: Comment on hypothesis-testing approaches to setting cutoff values for fit indexes and dangers in overgeneralizing Hu and Bentler's (1999) findings. Structural equation modeling, 11(3), 320-341.

McDonald-Miszczak, L., Hunter, M. A., \& Hultsch, D. F. (1994). Adult age differences in predicting memory performance: The effects of normative information and task experience. Canadian Journal of Experimental Psychology, 48(1), 95. 
McKay, C., Casey, J. E., Wertheimer, J., \& Fichtenberg, N. L. (2007). Reliability and validity of the RBANS in a traumatic brain injured sample. Archives of Clinical Neuropsychology, 22(1), 91-98.

Meeks, J. T., Hicks, J. L., \& Marsh, R. L. (2007). Metacognitive awareness of eventbased prospective memory. Consciousness and Cognition, 16(4), 997-1004.

Meier, B., von Wartburg, P., Matter, S., Rothen, N., \& Reber, R. (2011). Performance predictions improve prospective memory and influence retrieval experience. Canadian Journal of Experimental Psychology, 65(1), 12.

Muthén, B. O. (1991). Multilevel factor analysis of class and student achievement components. Journal of Educational Measurement, 28(4), 338-354.

Muthén, L. K., \& Muthén, B. O. (1998 - 2010). Mplus User's Guide (6 ${ }^{\text {th }}$ Ed). Los Angeles, CA: Muthén \& Muthén.

Randolph, C., Tierney, M. C., Mohr, E., \& Chase, T. N. (1998). The Repeatable Battery for the Assessment of Neuropsychological Status (RBANS): preliminary clinical validity. Journal of clinical and experimental neuropsychology, 20(3), 310-319.

Raykov, T., Tomer, A., \& Nesselroade, J. R. (1991). Reporting structural equation modeling results in Psychology and Aging: some proposed guidelines. Psychology and aging, 6(4), 499.

Roche, N. L., Fleming, J. M., \& Shum, D. H. (2002). Self-awareness of prospective memory failure in adults with traumatic brain injury. Brain injury, 16(11), 931-945. 
Rummel, J., Kuhlmann, B. G., \& Touron, D. R. (2013). Performance predictions affect attentional processes of event-based prospective memory. Consciousness and cognition, 22(3), 729-741.

Scheck, P., \& Nelson, T. O. (2005). Lack of pervasiveness of the underconfidencewith-practice effect: boundary conditions and an explanation via anchoring. Journal of Experimental Psychology: General, 134(1), 124.

Schnitzspahn, K. M., Ihle, A., Henry, J. D., Rendell, P. G., \& Kliegel, M. (2011a). The age-prospective memory-paradox: An exploration of possible mechanisms. International Psychogeriatrics, 23(04), 583-592.

Schnitzspahn, K. M., Zeintl, M., Jäger, T., \& Kliegel, M. (2011b). Metacognition in prospective memory: are performance predictions accurate? Canadian Journal of Experimental Psychology, 65(1), 19.

Schraw, G. \& Moshman, D. (1995). Metacognitive theories. Educational Psychology Review, 7(4), 351-371.

Schraw, G. (2009). A conceptual analysis of five measures of metacognitive monitoring. Metacognition and Learning, 4(1), 33-45.

Schraw, G., Crippen, K. J., \& Hartley, K. (2006). Promoting self-regulation in science education: Metacognition as part of a broader perspective on learning. Research in Science Education, 36, 111-139.

Serra, M. J., \& Ariel, R. (2014). People use the memory for past-test heuristic as an explicit cue for judgments of learning. Memory \& cognition, 42(8), 12601272. 
Serra, M. J., \& Dunlosky, J. (2005). Does retrieval fluency contribute to the underconfidence-with-practice effect?. Journal of Experimental Psychology: Learning, Memory, and Cognition, 31(6), 1258.

Smith, S. J., Souchay, C., \& Moulin, C. J. (2011). Metamemory and prospective memory in Parkinson's disease. Neuropsychology, 25(6), 734.

Souchay, C., Isingrini, M., \& Espagnet, L. (2000). Aging, episodic memory feelingof-knowing, and frontal functioning. Neuropsychology, 14(2), 299.

Strickland, L., Loft, S., Remington. R. W., \& Heathcote, A. (in press). Racing to remember: A theory of decision control in event-based prospective memory. Psychological Review.

Tauber, S. K., \& Dunlosky, J. (2015). Monitoring of learning at the category level when learning a natural concept: Will task experience improve its resolution?. Acta psychologica, 155, 8-18.

Tauber, S. K., \& Rhodes, M. G. (2012). Multiple bases for young and older adults' judgments of learning in multitrial learning. Psychology and Aging, 27(2), 474.

Toglia, J., \& Kirk, U. (2000). Understanding awareness deficits following brain injury. NeuroRehabilitation, 15(1), 57-70.

Tucker, L. R., \& Lewis, C. (1973). The reliability coefficient for maximum likelihood factor analysis. Psychometrika, 38, 1-10. 
Van Overschelde, J. P., Rawson, K. A., \& Dunlosky, J. (2004). Category norms: An updated and expanded version of the norms. Journal of Memory and Language, 50(3), 289-335.

Veenman, M. V., Van Hout-Wolters, B. H., \& Afflerbach, P. (2006). Metacognition and learning: Conceptual and methodological considerations. Metacognition and learning, 1(1), 3-14.

Whitebread, D., Coltman, P., Pasternak, D. P., Sangster, C., Grau, V., Bingham, S.,... \& Demetriou, D. (2009). The development of two observational tools for assessing metacognition and self-regulated learning in young children. Metacognition and Learning, 4(1), 63-85. 
Table 1. Regression equations testing concepts of anticipatory and emergent awareness, using prospective memory as example.

Regression Equation 1

Estimated Block 1 PM Prediction = Age + Education + Block 1 PM Performance

Unstandardised residuals (Observed Block 1 PM Prediction - Estimated Block 1 PM

Prediction) $=$ Anticipatory Awareness

Regression Equation 2

Step 1:

Estimated Block 2 PM Prediction = Age + Education + Block 2 PM Performance

Step 2:

Estimated Block 2 PM Prediction $=$ Age + Education + Block 2 PM Performance + Anticipatory Awareness

Unstandardised residuals (Observed Block 2 PM Prediction - Estimated Block 2 PM Prediction) $=$ Early Emergent Awareness

Regression Equation 3

Step 1:

Estimated Block 3 PM Prediction = Age + Education + Block 3 PM Performance

Step 2:

Estimated Block 3 PM Prediction = Age + Education + Block 3 PM Performance + Anticipatory Awareness

Step 3:

Estimated Block 3 PM Prediction = Age + Education + Block 3 PM Performance + Anticipatory Awareness + Early Emergent Awareness

Unstandardised residuals (Observed Block 3 PM Prediction - Estimated Block 3 PM Prediction) $=$ Late Emergent Awareness 
Table 2. Retrospective and Prospective memory accuracy, predictions, and prediction-performance discrepancies.

\begin{tabular}{|c|c|c|c|c|}
\hline \multicolumn{5}{|c|}{ Retrospective Memory $(\mathrm{N}=177)$} \\
\hline & Trial 1 & Trial 2 & Trial 3 & Trial 4 \\
\hline RM Accuracy & $50.34 \pm 13.81,0-100$ & $68.76 \pm 15.17,10-100$ & $77.80 \pm 14.59,30-100$ & $83.84 \pm 14.30,40-100$ \\
\hline RM Prediction & $55.38 \pm 13.14,0-80$ & $59.09 \pm 11.36,20-80$ & $66.34 \pm 13.78,20-100$ & $70.80 \pm 15.30,20-100$ \\
\hline $\begin{array}{l}\text { RM Prediction- } \\
\text { performance discrepancy }\end{array}$ & $4.97 \pm 17.41,-50-+70$ & $-9.77 \pm 14.97,-50-+60$ & $-11.54 \pm 14.08,-60-+30$ & $-13.10 \pm 13.92,-50-+3$ \\
\hline \multicolumn{5}{|l|}{ Prospective Memory $(\mathrm{N}=178)$} \\
\hline & Baseline (No PM task) & Block 1 & Block 2 & Block 3 \\
\hline PM Accuracy & - & $65.83 \pm 28.56,0-100$ & $63.70 \pm 28.51,0-100$ & $64.33 \pm 27.94,0-100$ \\
\hline PM Prediction & - & $72.80 \pm 18.18,0-100$ & $72.20 \pm 19.86,0-100$ & $73.90 \pm 19.66,0-100$ \\
\hline $\begin{array}{l}\text { PM Prediction- } \\
\text { performance discrepancy }\end{array}$ & - & $6.73 \pm 30.68,-70-+95$ & $8.51 \pm 31.10,-90-+95$ & $9.58 \pm 30.59,-70-+100$ \\
\hline Ongoing Task Accuracy & $96.04 \pm 3.10,81-100$ & $96.40 \pm 2.86,72-100$ & $97.03 \pm 2.15,83-100$ & $97.21 \pm 2.10,80-100$ \\
\hline Ongoing Task Prediction & $79.63 \pm 13.40,40-100$ & $77.05 \pm 14.12,30-100$ & $79.85 \pm 12.64,30-100$ & $80.88 \pm 11.91,50-100$ \\
\hline $\begin{array}{l}\text { Ongoing Task Prediction- } \\
\text { performance discrepancy }\end{array}$ & $-16.41 \pm 13.45,-60-+19$ & $-19.37 \pm 14.12,-67-+7$ & $-17.18 \pm 12.53,-66-+4$ & $-16.33 \pm 11.82,-48-+5$ \\
\hline Ongoing Task RT (ms) & $1482.11 \pm 305.24$ & $1633.09 \pm 373.45,953.66$ & $1472.60 \pm 320.50$ & $1405.12 \pm 301.52$ \\
\hline
\end{tabular}


$830.43-2338.07$

$-2638.21$

$933.87-2568.97$

$855.47-2444.52$

Values presented as Mean percentage \pm Standard deviation, Range; PM = Prospective memory; RM = Retrospective memory; Discrepancies calculated as

(Prediction - Performance), such that negative scores indicate underconfidence and positive overconfidence; RT = Response times. 
Table 3. Summary of hierarchical regression analysis for variables predicting retrospective memory predictions $(N=169)$

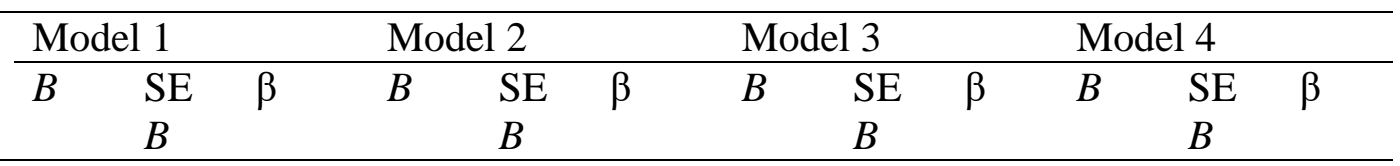

Trial 1

\begin{tabular}{lllllllllllll}
\hline Age & -.18 & .13 & -.11 & - & - & - & - & - & - & - & - & - \\
Education & .02 & .30 & .01 & - & - & - & - & - & - & - & - & - \\
RM & .15 & .07 & .15 & - & - & - & - & - & - & - & - & - \\
Performance & & & & & & & & & & & &
\end{tabular}

Trial 2

$\begin{array}{lllllll}\text { Age } & -.08 & .11 & -.06 & -.09 & .10 & -.06\end{array}$

$\begin{array}{lllllll}\text { Education } & .63 & .23 & .19^{+} & .64 & .22 & .19^{+}\end{array}$

RM

$\begin{array}{llllll}.27 & .05 & .36^{+} & .25 & .05 & .33^{+}\end{array}$

Performance

AA

$.30 \quad .06 \quad .34^{+}$

Trial 3

$\begin{array}{lllllllllllll}\text { Age } & .10 & .12 & .05 & .09 & .12 & .05 & .09 & .09 & .05 & - & - & - \\ \text { Education } & .66 & .27 & .16^{*} & .66 & .26 & .17^{*} & .69 & .20 & .17^{+}- & - & - \\ \text {RM } & .46 & .07 & .49^{+} & .45 & .06 & .48^{+} & .42 & .05 & .44^{+}- & - & - \\ \text {Performance } & & & & & & & & & & & & \\ \text { AA } & - & - & - & .24 & .07 & .22^{+} & .24 & .05 & .22^{+}- & - & - \\ \text {EEA } & - & - & - & - & - & - & .77 & .07 & .52^{+}- & - & -\end{array}$

Trial 4

\begin{tabular}{lcccccccccccc}
\hline Age & -.08 & .13 & -.04 & -.08 & .13 & -.04 & -.08 & .11 & -.04 & -.11 & .09 & -.06 \\
Education & .60 & .29 & $.14^{*}$ & .61 & .29 & $.14^{*}$ & .64 & .24 & $.14^{+}$ & .69 & .21 & $.15^{+}$ \\
RM & .55 & .07 & $.52^{+}$ & .54 & .07 & $.50^{+}$ & .50 & .06 & $.47^{+}$ & .42 & .05 & $.39^{+}$ \\
Performance & & & & & & & & & & & & \\
AA & - & - & - & .22 & .08 & $.18^{+}$ & .22 & .06 & $.19^{+}$ & .23 & .05 & $.19^{+}$ \\
EEA & - & - & - & - & - & - & .70 & .09 & $.43^{+}$ & .71 & .07 & $.44^{+}$ \\
MEA & - & - & - & - & - & - & - & - & - & .63 & .08 & $.36^{+}$ \\
\hline
\end{tabular}

${ }^{*} \mathrm{p}<.05 ;{ }^{+} \mathrm{p}<.01 ; \mathrm{SE}=$ Standard Error; RM = Retrospective Memory; AA = Anticipatory Awareness; EEA = Early Emergent Awareness; MEA = Mid Emergent Awareness. 
Table 4. Summary of stepwise multiple regression analysis for variables predicting PM predictions $(N=173)$.

Model $1 \quad$ Model $2 \quad$ Model 3

\begin{tabular}{lllllllll}
\hline$B$ & SE $B$ & $\beta$ & $B$ & SE $B$ & $\beta$ & $B$ & SE $B$ & $\beta$
\end{tabular}

\section{Block 1}

\begin{tabular}{|c|c|c|c|c|c|c|c|c|c|}
\hline Age & -.03 & .18 & -.01 & - & - & - & - & - & - \\
\hline Education & .39 & .40 & .07 & - & - & - & - & - & - \\
\hline PM Performance & .12 & .05 & $.18^{\dagger}$ & - & - & - & - & - & - \\
\hline
\end{tabular}

Block 2

\begin{tabular}{lccccccccc}
\hline Age & -.01 & .20 & -.004 & -.02 & .15 & -.01 & - & - & - \\
Education & .54 & .45 & .09 & .58 & .35 & .10 & - & - & - \\
PM Performance & .13 & .05 & $.19^{\dagger}$ & .12 & .04 & $.17^{+}$ & - & - & - \\
AA & - & - & - & .69 & .07 & $.62^{+}$ & - & - & - \\
\hline
\end{tabular}

Block 3

\begin{tabular}{|c|c|c|c|c|c|c|c|c|c|}
\hline Age & .01 & .20 & 0 & 0 & .15 & 0 & -.04 & .11 & -.02 \\
\hline Education & .19 & .45 & .03 & .25 & .35 & .04 & .44 & .26 & .08 \\
\hline PM Performance & .14 & .06 & $.20^{\dagger}$ & .12 & .04 & $.17^{+}$ & .04 & .03 & .05 \\
\hline AA & - & - & - & .67 & .07 & $.61^{+}$ & .68 & .05 & $.62 \ddagger$ \\
\hline EEA & - & - & - & - & - & - & .70 & .06 & $.53^{\ddagger}$ \\
\hline
\end{tabular}


Supplementary Table 1. Growth curve model building for retrospective memory.

\begin{tabular}{|c|c|c|c|c|c|c|c|c|c|c|c|c|c|c|c|c|}
\hline \multirow[t]{3}{*}{ Model } & \multirow[t]{3}{*}{$\chi^{2}$} & \multirow[t]{3}{*}{$d f$} & \multirow[t]{3}{*}{$p$} & \multirow[t]{3}{*}{$\chi^{2} / \mathrm{df}$} & \multirow[t]{3}{*}{ AIC } & \multirow{3}{*}{$\begin{array}{l}\text { Parsimony } \\
\text { ratio }\end{array}$} & \multirow[t]{3}{*}{ RMSEA } & \multirow[t]{3}{*}{ CFI } & \multirow[t]{3}{*}{ TLI } & \multirow[t]{3}{*}{ SRMR } & \multicolumn{6}{|c|}{ Growth parameter estimates } \\
\hline & & & & & & & & & & & \multicolumn{2}{|c|}{ Intercept } & \multicolumn{2}{|c|}{$\underline{\text { Slope }}$} & \multicolumn{2}{|c|}{ Quadratic } \\
\hline & & & & & & & & & & & Mean & Var & Mean & Var & Mean & Var \\
\hline \multicolumn{17}{|c|}{ Retrospective memory performance $(\mathrm{N}=177)$} \\
\hline Intercept only & 499.96 & 8 & $<.001$ & 62.50 & 6012.80 & .89 & $\begin{array}{c}.59 \\
(.55-.63)\end{array}$ & .00 & -.31 & 1.26 & $76.48^{\ddagger}$ & $137.18^{\ddagger}$ & - & - & - & - \\
\hline Linear change & 87.36 & 5 & 0 & 17.47 & 5606.20 & .50 & $\begin{array}{c}.31 \\
(.25-.36)\end{array}$ & .71 & .65 & .12 & $55.16^{\ddagger}$ & $84.31^{\ddagger}$ & $30.72^{\ddagger}$ & 28.60 & - & - \\
\hline $\begin{array}{l}\text { Quadratic } \\
\text { change }\end{array}$ & 5.15 & 1 & .023 & 5.15 & 5531.99 & .10 & $\begin{array}{c}.15 \\
(.05-.29)\end{array}$ & .99 & .91 & .03 & $50.73^{\ddagger}$ & $102.07^{\dagger}$ & $59.05^{\ddagger}$ & 625.33 & $-26.33^{\ddagger}$ & 181.40 \\
\hline Logarithmic* & 11.79 & 5 & .038 & 2.36 & 5530.63 & .50 & $\begin{array}{c}.00 \\
(.02-.15)\end{array}$ & .98 & .97 & .08 & $50.97^{\ddagger}$ & $106.76^{\ddagger}$ & $33.40^{\ddagger}$ & $100.37^{\ddagger}$ & - & - \\
\hline $\begin{array}{l}\text { Approximately } \\
\text { Logarithmic }\end{array}$ & 8.16 & 4 & .086 & 2.04 & 5529.00 & .40 & $\begin{array}{c}.08 \\
(0-.15)\end{array}$ & .99 & .98 & .09 & $50.45^{\ddagger}$ & $111.90^{\ddagger}$ & $33.74^{\ddagger}$ & $111.81^{\ddagger}$ & - & - \\
\hline $\begin{array}{l}\text { Time metric } \\
\text { freely estimated } \\
(0, *, *, 1)\end{array}$ & 6.35 & 3 & .096 & 2.12 & 5529.20 & .30 & $\begin{array}{c}.08 \\
(0-.17)\end{array}$ & .99 & .98 & .10 & $50.27^{\ddagger}$ & $111.98^{\ddagger}$ & $33.50^{\ddagger}$ & $109.94^{\ddagger}$ & - & - \\
\hline
\end{tabular}

$\underline{\text { Retrospective memory prediction }(\mathrm{N}=177)}$ 


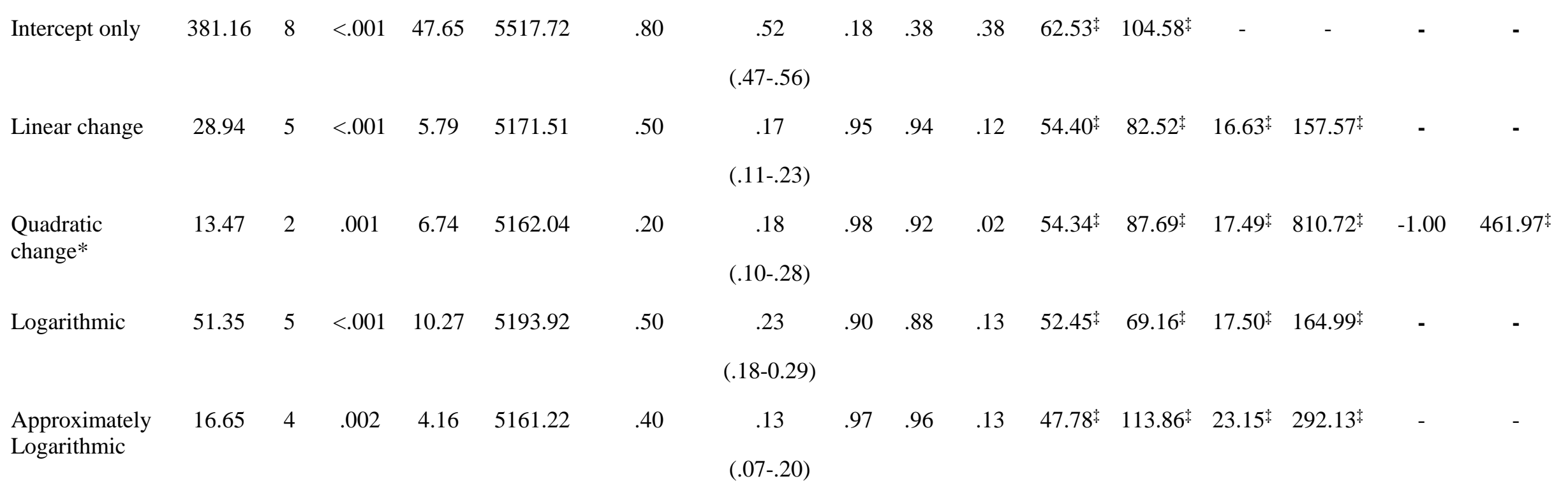

Combination of Retrospective memory prediction and performance $(\mathrm{N}=177)$

$\begin{array}{lllllllllllllll}\text { Combined } & 56.61 & 17 & <.001 & 3.33 & 10589.72 & .47 & .12 & .95 & .92 & .07 & -\end{array}$

$(.08-.15)$

*Best fitting models for each respective section; ${ }^{\dagger} p<.05 ;{ }^{\ddagger} p<.01$; $\mathrm{df}=$ Degrees of freedom; AIC = Akaike information criterion; RMSEA = Root mean

square error of approximation; CFI = Comparative fit index; TLI = Tucker-Lewis index; SRMR = Standardised root mean square residual; SE = Standard

error; Parsimony ratio is defined as $\mathrm{df} /[.5 \mathrm{k}(\mathrm{k}+1)]$ where $\mathrm{k}$ is the number of observed variables. 
Supplementary Table 2. Growth curve model building for prospective memory.

\begin{tabular}{|c|c|c|c|c|c|c|c|c|c|c|c|c|c|c|}
\hline \multirow[t]{3}{*}{ Model } & \multirow[t]{3}{*}{$\chi^{2}$} & \multirow[t]{3}{*}{$d f$} & \multirow[t]{3}{*}{$\chi^{2 / \mathrm{df}}$} & \multirow[t]{3}{*}{$P$} & \multirow[t]{3}{*}{ AIC } & \multirow{3}{*}{$\begin{array}{c}\text { Parsimony } \\
\text { ratio }\end{array}$} & \multirow[t]{3}{*}{ RMSEA } & \multirow[t]{3}{*}{ CFI } & \multirow[t]{3}{*}{ TLI } & \multirow[t]{3}{*}{ SRMR } & \multicolumn{4}{|c|}{ Growth parameter estimates } \\
\hline & & & & & & & & & & & \multicolumn{2}{|c|}{$\underline{\text { Intercept }}$} & \multicolumn{2}{|c|}{$\underline{\text { Slope }}$} \\
\hline & & & & & & & & & & & Mean & Var & Mean & Var \\
\hline \multicolumn{15}{|c|}{ Prospective memory performance $(\mathrm{N}=178)$} \\
\hline Intercept only* & 3.53 & 4 & .88 & .47 & 4869.70 & .67 & $0(0-.11)$ & 1.00 & 1.00 & .06 & $64.51^{\ddagger}$ & $524.99^{\ddagger}$ & - & - \\
\hline Linear change & .80 & 1 & .80 & .37 & 4872.96 & .17 & $0(0-.19)$ & 1.00 & 1.00 & .01 & $65.24^{\ddagger}$ & $446.46^{\ddagger}$ & -1.21 & 18.54 \\
\hline \multicolumn{15}{|c|}{ Prospective memory prediction $(\mathrm{N}=178)$} \\
\hline Intercept only & 21.38 & 4 & 5.35 & $<.001$ & 4362.38 & .67 & $.16(.10-.22)$ & .95 & .96 & .22 & $73.00^{\ddagger}$ & $288.49^{\ddagger}$ & - & - \\
\hline Linear change* & 2.74 & 2 & 1.37 & .25 & 4347.73 & .33 & $.05(0-.16)$ & 1.00 & 1.00 & .05 & $72.47^{\ddagger}$ & $248.80^{\ddagger}$ & 1.44 & $153.61^{\ddagger}$ \\
\hline \multicolumn{15}{|c|}{ Combination of Prospective memory prediction and performance $(\mathrm{N}=178)$} \\
\hline Combined & 19.71 & 13 & 1.52 & .10 & 9209.06 & .62 & $.05(0-.10)$ & .99 & .99 & .06 & - & - & - & - \\
\hline
\end{tabular}

